\title{
High-throughput sequencing of murine immunoglobulin heavy chain repertoires using single side unique molecular identifiers on an Ion Torrent PGM
}

\author{
Jean-Philippe Bürckert ${ }^{1}$, William J. Faison ${ }^{1}$, Danielle E. Mustin ${ }^{1}$, Axel R.S.X. \\ Dubois $^{1}$, Regina Sinner ${ }^{1}$, Oliver Hunewald ${ }^{1}$, Anke Wienecke-Baldacchino ${ }^{1}$, Anne \\ Brieger $^{1, *}$ and Claude P. Muller ${ }^{1, *}$ \\ ${ }^{1}$ Department of Infection and Immunity, Luxembourg Institute of Health, Esch-sur-Alzette, Luxembourg \\ * These authors share senior authorship \\ Correspondence to: Jean-Philippe Bürckert, email: jean-philippe.buerckert@lih.lu \\ Claude P. Muller, email: claude.muller@lih.lu
}

Keywords: high-throughput sequencing; murine IG repertoire; unique molecular barcoding; database benchmarking; IMGT; Immunology

Received: August 31, 2017

Accepted: May 07, 2018

Published: July 13, 2018

Copyright: Bürckert et al. This is an open-access article distributed under the terms of the Creative Commons Attribution License 3.0 (CC BY 3.0), which permits unrestricted use, distribution, and reproduction in any medium, provided the original author and source are credited.

\section{ABSTRACT}

With the advent of high-throughput sequencing (HTS), profiling immunoglobulin (IG) repertoires has become an essential part of immunological research. Advances in sequencing technology enable the IonTorrent Personal Genome Machine (PGM) to cover the full-length of IG mRNA transcripts. Nucleotide insertions and deletions (indels) are the dominant errors of the PGM sequencing platform and can critically influence IG repertoire assessments. Here, we present a PGM-tailored IG repertoire sequencing approach combining error correction through unique molecular identifier (UID) barcoding and indel detection through ImMunoGeneTics (IMGT), the most commonly used sequence alignment database for IG sequences. Using artificially falsified sequences for benchmarking, we found that IMGT's underlying algorithms efficiently detect $\mathbf{9 8 \%}$ of the introduced indels. Undetected indels are either located at the end of the sequences or produce masked frameshifts with an insertion and deletion in close proximity. The complementary determining regions 3 (CDR3s) are returned correct for up to 3 insertions or 3 deletions through conservative culling. We further show, that our PGM-tailored unique molecular identifiers result in highly accurate HTS data if combined with the presented processing strategy. In this regard, considering sequences with at least two copies from datasets with UID families of minimum 3 reads result in correct sequences with over $99 \%$ confidence. Finally, we show that the protocol can readily be used to generate homogenous datasets for bulk sequencing of murine bone marrow samples. Taken together, this approach will help to establish benchtop-scale sequencing of IG heavy chain transcripts in the field of IG repertoire research.

\section{INTRODUCTION}

The diversity of the immunoglobulin (IG) repertoire is the key feature of the adaptive immune system, enabling it to theoretically combat every possible antigen encountered during an individual's lifetime [1]. With the development of high-throughput sequencing (HTS) it became possible to analyze the IG repertoire at high depth [2-6]. Studies, almost a decade ago, established Roche's 454 sequencer as the first tool of choice for exhaustive characterization of IG repertoires due to its superior read-length [7]. More recently, Illumina's MiSeq and 
HiSeq sequencers as well as the Ion Torrent Personal Genome Machine (PGM, Thermo Fisher Scientific) provided improved sequencing technologies which can reach across the full $\mathrm{V}(\mathrm{D}) \mathrm{J}$ nucleotide sequence [8]. The different technologies of the sequencers result each in their specific error-rates and -types [7, 9-15]. Illumina's optical sequencing produces mostly nucleotide (nt) transversions and transitions, which can be corrected by building consensus sequences [16]. The 454's pyrosequencing chemistry and the PGMs semiconductor technique mainly introduce homopolymer repeats resulting in insertions and deletions of bases, which can be corrected by gene segment-wise reference alignment [17].

Most sequencing approaches use IG isotype specific constant $(\mathrm{C})$ region primers to translate IG heavy-chain (IGH) (m)RNA into cDNA, which are subsequently amplified using a set of V-region specific primers in a multiplex PCR approach. However, this can result in skewed repertoire assessments due to biased PCR efficacy $[8,14,18]$. In addition, sequencing errors can falsify somatic hypermutation profiles, VDJ germline gene assignment and clonal grouping [8, 19]. Unique molecular identifiers (UID) which tag individual RNA molecules at cDNA transcription level have been used to obtain an unbiased view on the IG repertoire [20-23]. This method also allows thorough error correction by building consensus sequences, albeit at the cost of sequencing depth. In all cases, complex bioinformatic approaches are necessary to perform raw-read processing [24]. Subsequent alignments to germline genes to assign VDJ family genes are in general conducted using the V-QUEST or HighV-QUEST tools available at the ImMunoGeneTics (IMGT) database, which applies an error correction algorithm for insertions and deletions in the process [25, 26].

After the initial proof-of-concept studies, the use of animal models to study the IG repertoire dynamics has been largely ignored $[4,6]$. One major factor being the lack of a suitable IGH V-region primer set comparable to BIOMED-2, developed for the human IG repertoire [27]. Yet, animal models offer advantages over human studies, as they are not limited to peripheral blood and have a lower B cell diversity [28-30]. As IMGT provides germline repertoires for various species, we chose to develop a method to profile the IG repertoire of Balb/C mice, one of the most commonly used animal models.

In the present study, the performance of the PGM sequencing platform together with the IMGT HighVQUEST tool for the assessment of murine IGH repertoires is evaluated. In this context, several novel aspects are examined: first, the IMGT's indel detection and correction algorithm is benchmarked with a set of artificially falsified sequences. Second, a 16-nucleotide single side UID (ssUID) barcoding technique tailored to the PGM sequencing chemistry is introduced together with a swift 1-day library preparation protocol. Third, the PGM's error-rate for sequencing murine IG transcripts with our barcoding strategy and customized data processing is determined.

\section{RESULTS}

\section{Reference sequences}

A set of 7 monoclonal Balb/C mouse hybridoma cell lines was used to investigate the distribution and influence of insertions and deletions (indels) produced by the IonTorrent PGM sequencing technology on murine IGH repertoire sequencing (Figure 1). Reference sequences were obtained from Sanger sequenced cDNA transcripts of monoclonal hybridoma RNA subsequently annotated, with native germline sequences identified (see Supplementary Table 1) and translated into amino acids by IMGT V-QUEST.

\section{Distribution of artificial insertions and deletions}

To investigate the influence of indels on IMGT HighV-QUEST processing of an IGH sequence, we generated a benchmark dataset from the reference sequences that contained artificially introduced indels at random positions (Supplementary Table 2). To cover each position within a $300 \mathrm{nt}$ sequence with minimum $90 \%$ certainty, at least 2398 erroneous variants are required [31]. Therefore, we generated 2500 artificial, randomly flawed sequences for each permutation of 0-3 insertions and/or deletions (indels, annotated as i1d0, i0d1, ild1 ... $\mathrm{i} 3 \mathrm{~d} 3$ ), resulting in a total of 37500 artificial sequences per original hybridoma sequence with indels ranging from 1 to 6 events. Indels were homogenously present as determined by graphical reference alignment (Figure 2A). Uncovered positions resulted from indels within homopolymer stretches which were always assigned to the beginning of such a nucleotide repeat region (Figure 2B).

\section{IMGT HighV-QUEST VDJ nucleotide error detection}

As each sequence of the benchmark system contained indel errors, all sequences marked by IMGT HighV-QUEST as productive were falsely categorized as error free. In general, IMGT HighV-QUEST correctly recognized $97.9 \%( \pm 2.9 \%)$ of the introduced indels over all datasets and categorized the sequences then either as productive with detected indels, unproductive or unknown (Figure 2C). Interestingly, only the sets with one insertion and/or deletion (i1d0, i0d1 and i1d1) exhibited elevated numbers of unrecognized indels. For these IMGT HighVQUEST falsely returned $8 \%( \pm 1.8 \%)$ of the sequences 
as productive, whereas for all other datasets it was only $0.7 \%( \pm 0.4 \%)$. Such undetected indels were found at the beginning and the end of the sequence or across the whole sequence for ild1 datasets due to indels in close proximity to each other masking the frame-shifts (Figure
2D, Figure 3, supplementary Figure 1 and 2). The number of unproductive sequences increased with the number of indel events, regardless of their composition. Accordingly, the number of productive sequences with detected indels decreased. Less than $50 \%$ of sequences with more than
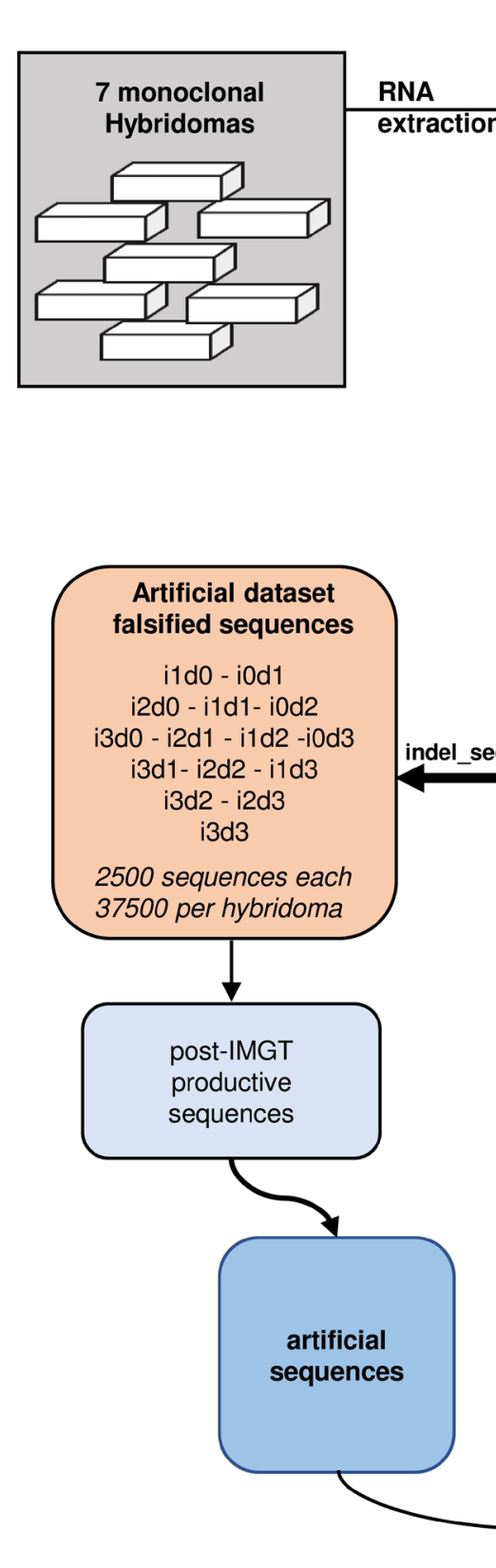
$3 d 3$

l dataset sequences

d0 - i0d1

i1d2 -i0d3 i2d3 productive equences

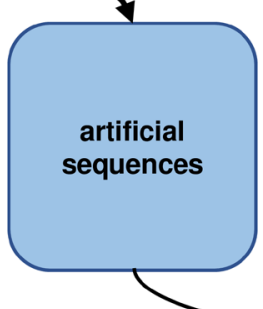

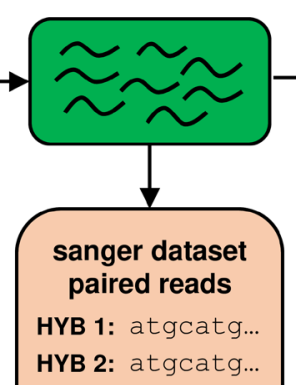
HYB 3: atgcatg... HYB 4: atgcatg... HYB 5: atgcatg... HYB 6: atgcatg... HYB 7: atgcatg...
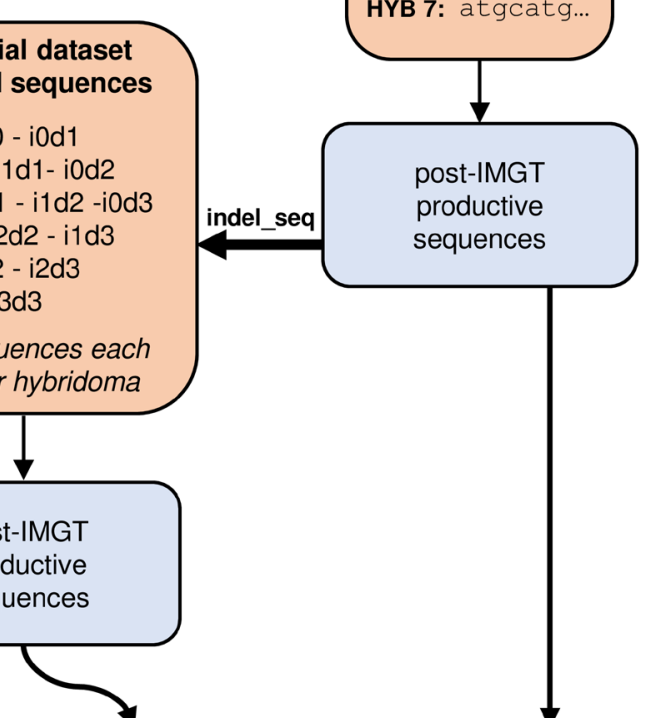

three step HTS, library preparation (12.

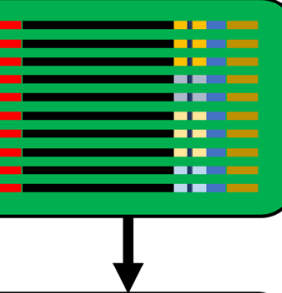

HTS dataset Untrimmed raw reads

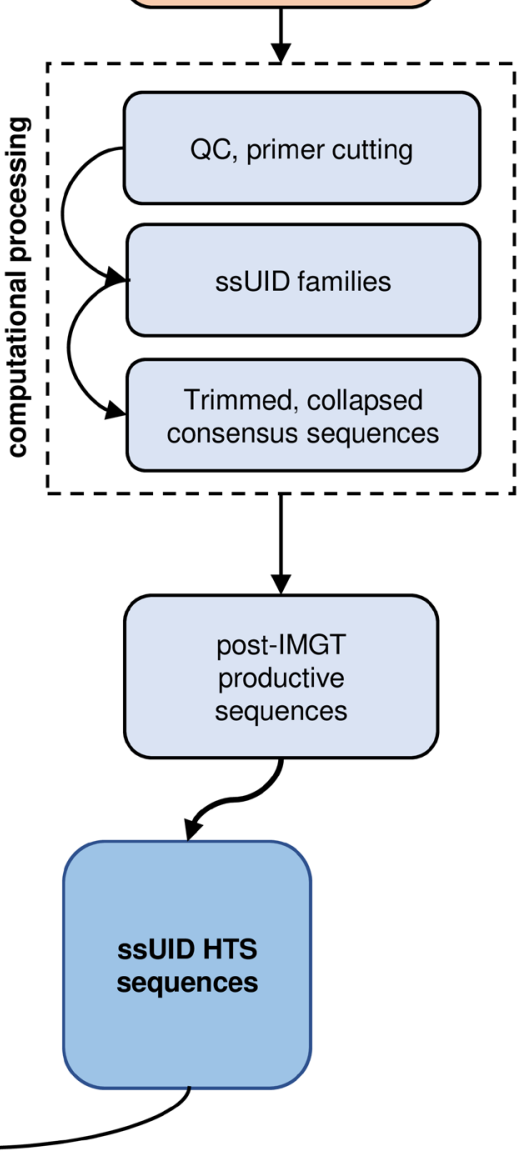

alignment and error detection

Figure 1: Study design. RNA was extracted from 7 monoclonal hybridoma cell lines and reverse transcribed into cDNA. cDNA sequences were determined by Sanger sequencing and submitted to IMGT V-QUEST to determine reference sequences. Reference sequences were artificially falsified using the indel_seq program, introducing up to 3 insertions and 3 deletions. 2500 artificial sequences were generated for each permutation and hybridoma and processed by IMGT HighV-QUEST. Post-IMGT HighV-QUEST sequences were aligned to the references to determine error detection and correction. RNA was also used to generate high-throughput sequencing (HTS) libraries in a three-step library preparation protocol. Single side unique identifiers (ssUID) were introduced during reverse transcription to tag each RNA molecule individually (see also supplementary Figure 3). Libraries were sequenced on an Ion Torrent PGM sequencer with all quality trimming options disabled in the Torrent Suite software. Untrimmed raw sequences were processed with a custom-made bioinformatics pipeline generating consensus sequences per UID family. Collapsed consensus sequences were submitted to IMGT HighVQUEST and post-IMGT HighV-QUEST sequences aligned to the reference sequences to determine error detection and correction. 
Table 1: HTS hybridoma datasets pre-IMGT

\begin{tabular}{|l|l|l|l|l|}
\hline Set & Chip & $\begin{array}{l}\text { reads with } \\
\text { MID }\end{array}$ & $\begin{array}{l}\text { reads with } \\
\text { primer } \\
\text { UID }\end{array}$ & $\begin{array}{l}\text { consensus } \\
\text { sequences }\end{array}$ \\
\hline HYB1 & A & 207,753 & 206,929 & 4,159 \\
\hline HYB2 & A & 147,634 & 146,010 & 7,760 \\
\hline HYB3 & A & 222,929 & 222,100 & 1,431 \\
\hline HYB4 & A & 882,242 & 877,823 & 16,643 \\
\hline HYB5 & B & 747,827 & 733,258 & 7,319 \\
\hline HYB6 & B & 743,465 & 739,854 & 47,169 \\
\hline HYB7 & B & 204,348 & 201,619 & 5,426 \\
\hline BM1 & C & 679,600 & 581,983 & 37,877 \\
\hline BM2 & C & 592,044 & 533,839 & 37,388 \\
\hline BM3 & C & 566,441 & 517,149 & 32,748 \\
\hline BM4 & C & 722,267 & 643,847 & 38,635 \\
\hline
\end{tabular}

3 indels, were retained. Indels were homogenously distributed in the uncorrected productive sequences with detected errors until about $4 / 5^{\text {th }}$ of the sequence lengths while the opposite is true for the uncorrected unproductive sequences (Figure 2D, Figure 3 and supplementary Figure 2). This section of the sequence coincides with the IMGT IGH junction which encodes for the CDR3 [32]. Accordingly, upon detecting an indel in the IGH junction, IMGT HighV-QUEST categorized the sequence as unproductive and no corrective attempts were made.

\section{IMGT HighV-QUEST VDJ nucleotide error correction}

Upon detection of an indel, IMGT HighV-QUEST tries to correct it by alignment to its closest germline. The efficacy of this process was investigated by aligning the sequences with detected indels to determine the number of correctly resolved sequences (Figure 3, Figure 4 and
A

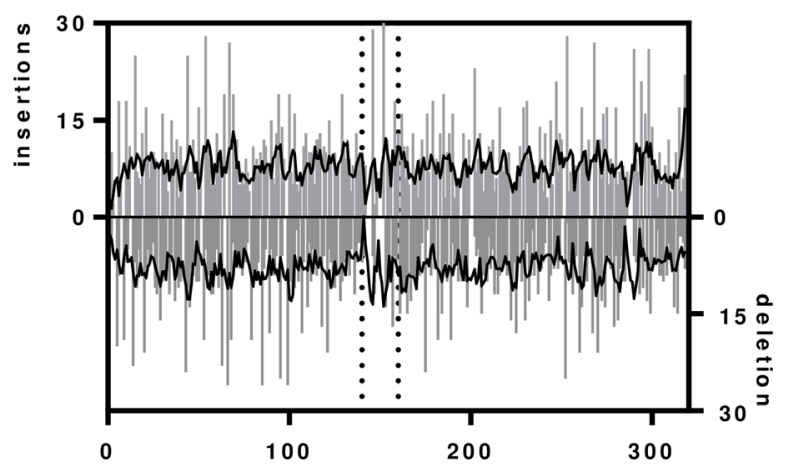

C

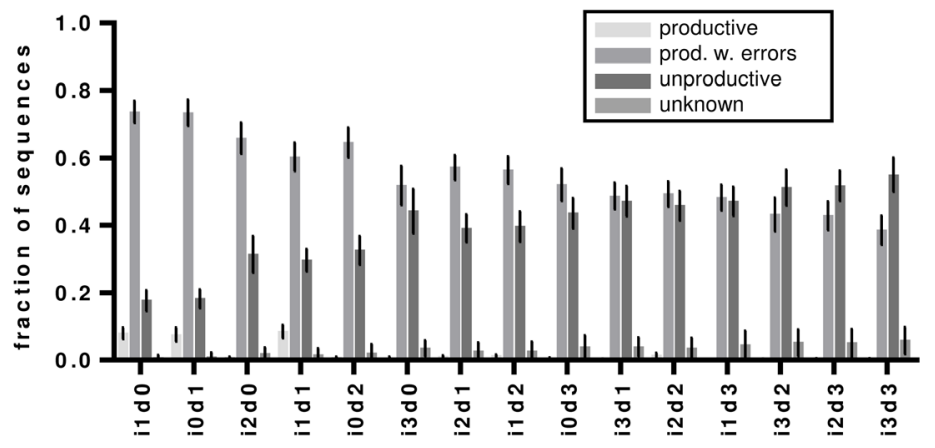

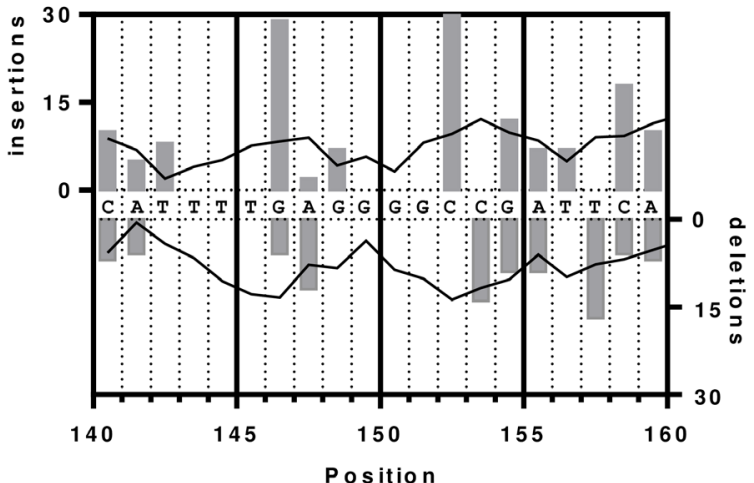

D

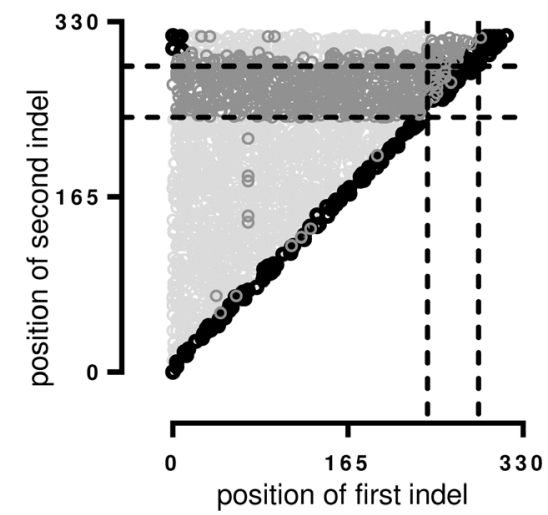

Figure 2: Indels in the artificial dataset. A. Insertion and deletion events displayed as determined by graphical alignments of the reference sequence to the $\mathrm{i} 1 \mathrm{~d} 0$ and i0d1 dataset of hybridoma 1. Grey bars represent the actual detected indel and the black line presents the moving average over 4 neighbors. The dotted vertical lines represent the segment that is magnified in B. to visualize the problem of determining the position of indels within homopolymer repeats. C. Indel detection rates by IMGT HighV-QUEST processing shown as bar chart with error bars indicating the SD over all 7 datasets. D. Visualization of frame-shift masking indel proximity in Hybridoma 1 ild1 dataset. The nt positions of the first and second indel before correction are shown as scatterplot. Dotted lines indicate the position of the IMGT IGH junction. Productive sequences with detected indels are shown in light grey, unproductive sequences are shown in dark grey. Sequences without detected errors are shown in black. The remaining i1d1 indel proximity graphs are shown in the supplementary Figure 1. 
Table 2: HTS hybridoma hybridoma datasets classifications by IMGT HighV-QUEST

\begin{tabular}{|l|l|l|l|l|l|l|l|l|}
\hline Set & prod. seq. & $\%$ & prod. w. det. indel & $\%$ & unprod & \% & unknown/ else & $\%$ \\
\hline HYB1 & 3,328 & $79.6 \%$ & 622 & $14.9 \%$ & 127 & $3.0 \%$ & 102 & $2.4 \%$ \\
\hline HYB2 & 4,866 & $62.7 \%$ & 2,449 & $31.6 \%$ & 250 & $3.2 \%$ & 195 & $2.5 \%$ \\
\hline HYB3 & 381 & $26.6 \%$ & 62 & $4.3 \%$ & 984 & $68.8 \%$ & 4 & $0.3 \%$ \\
\hline HYB4 & 13,515 & $81.2 \%$ & 2,215 & $13.3 \%$ & 329 & $2.0 \%$ & 584 & $3.5 \%$ \\
\hline HYB5 & 6,697 & $91.5 \%$ & 281 & $3.8 \%$ & 51 & $0.7 \%$ & 290 & $4.0 \%$ \\
\hline HYB6 & 43,767 & $92.8 \%$ & 3,009 & $6.4 \%$ & 287 & $0.6 \%$ & 106 & $0.2 \%$ \\
\hline HYB7 & 5,216 & $96.1 \%$ & 111 & $2.0 \%$ & 15 & $0.3 \%$ & 84 & $1.5 \%$ \\
\hline Mean & 11,110 & $75.8 \%$ & 1,250 & $10.9 \%$ & 292 & $11.2 \%$ & 195 & $2.1 \%$ \\
\hline SD & 13,842 & $22.6 \%$ & 1,165 & $9.6 \%$ & 303 & $23.5 \%$ & 180 & $1.4 \%$ \\
\hline
\end{tabular}

Table 3: Ambiguous nt in HTS hybridoma datasets

\begin{tabular}{|l|l|l|l|l|l|l|l|l|}
\hline & HYB1 & HYB2 & HYB4 & HYB5 & HYB6 & HYB7 & Mean & SD \\
\hline Amb nt & 26 & 135 & 97 & 90 & 2289 & 148 & 464 & 817 \\
\hline$\%$ & 0.8 & 2.6 & 0.7 & 1.3 & 5.2 & 2.8 & 2.2 & 1.6 \\
\hline
\end{tabular}

supplementary Figure 2). A thorough error reduction was observed for up to three insertion errors in datasets without deletions, returning $87 \% \pm 3.2 \%$ (i1d 0 ), $72 \% \pm 5.5 \%$ (i2 $\mathrm{d} 0)$ and $56 \% \pm 7.0 \%$ (i3d 0$)$ of productive sequences as correct (Figure 4). Within these sequences indels that were not corrected by the IMGT HighV-QUEST were mainly found at the beginning and end of the sequence (Figure $3 \mathrm{~A}, 3 \mathrm{D}, 3 \mathrm{E})$. In the case of deletions, the IMGT HighVQUEST correction introduced a gap for the missing nucleotide as the original nucleotide was unknown. Consequently, the number of correct sequences found in datasets with mixed insertions and deletions is very low (i1d1: $1 \% \pm 0.3 \%, \mathrm{i} 2 \mathrm{~d} 1: 2 \% \pm 0.3 \%, \mathrm{i} 3 \mathrm{~d} 1: 2 \% \pm 0.6 \%$, $\mathrm{i} 2 \mathrm{~d} 2$ and $\mathrm{i} 3 \mathrm{~d} 2<1 \%$ ). Nevertheless, in these datasets, the insertions within the sequences were always reduced (Figure 3C and supplementary Figure 2). No correct sequence could be identified in deletion-only datasets (Figure 4).

\section{IMGT HighV-QUEST VDJ amino acid error correction}

Theoretically, translated amino acids are less influenced by sequencing errors because of the redundancy of the genetic code. Thus, most amino acid translations were returned correctly in the case of insertion-only datasets and with slightly higher numbers compared to the nucleotide datasets (mean correct amino acid sequences for i1d0: $89 \% \pm 2.9 \%$, i $2 \mathrm{~d} 0: 76 \% \pm 4.7 \%$, i3d0: $61 \% \pm$ $6.5 \%$, Figure 4 ). Higher numbers of correct translations were observed in mixed indel datasets than for the corresponding nucleotide datasets (i1d $1: 3 \% \pm 0.7 \%, \mathrm{i} 2 \mathrm{~d} 1$ : $4 \% \pm 0.6 \%, \mathrm{i} 3 \mathrm{~d} 1: 4 \% \pm 0.8 \%, \mathrm{i} 2 \mathrm{~d} 2$ and $\mathrm{i} 3 \mathrm{~d} 2<1 \%$, Figure 4). Interestingly, some amino acid translations were found to be correct for the i0d 1 datasets $(1 \% \pm 0.5 \%$, Figure 4$)$.
Deletion-affected datasets were usually returned with the wrong amino acid sequence by the underlying algorithm. During IMGT HighV-QUEST processing, nucleotide deletions rendered the whole codon triplet elusive and were translated as gaps in the amino acid sequence.

Remarkably, the CDR3 proved to be protected chiefly from insertions and deletions through a more conservative correction approach of the IMGT HighVQUEST algorithm for this part of the sequence. As mentioned above, detected indels within the IGH junction, and thus the CDR3, corrupted the entire sequence as unproductive (Figure 3 and supplementary Figure 2). Culling attempts by IMGT HighV-QUEST turned out to be largely successful $(100 \%$ correct CDR3s for up to 3 insertions or 3 deletions). Even for the $\mathrm{i} 3 \mathrm{~d} 3$ indel permutation, IMGT HighV-QUEST returned 78\% \pm $4.3 \%$ correct CDR3s (Figure 4), by removing all those sequences where indels were detected in the CDR3 encoding nucleotides. Datasets with simultaneous insertions and deletions showed in general lower numbers of correct CDR3 sequences (range 78-97\%). This resulted from sequences where indels were introduced in close proximity of each other, producing no detectable frameshift within the IGH junction (Figure 2D). While invisible for the IMGT HighV-QUEST algorithm, they were observed as variants of the correct CDR3 amino acid sequence.

Taken together the above data show, that IMGT HighV-QUEST processing exhibits adequate detection of indels through frame-shifts in mouse IGH nt sequences. Consequently, frame-shift masking error compositions cannot be detected and result in amino acid changes in the translations. IMGT's HighV-QUEST indel correction proved to be reliable for single insertions. However, the impossibility to correct for deletions and larger indel permutations makes consideration of sequences 


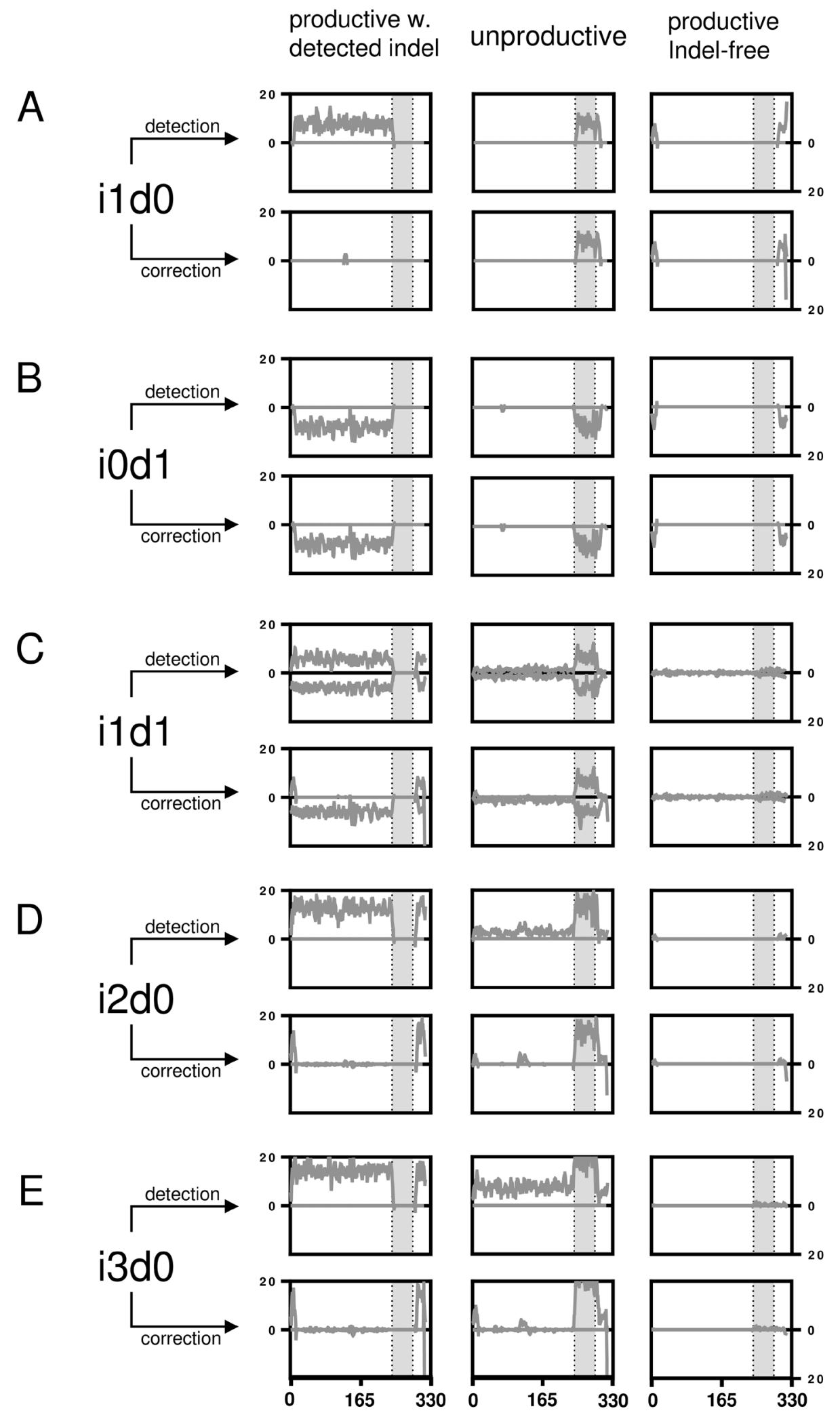

Figure 3: Artificial indel set alignments. Number of indels are shown per nucleotide position before and after IMGT HighV-QUEST error correction for artificially falsified Hybridoma 1 sequences separated by productivity as returned by IMGT HighV-QUEST. A. The number of indels for the ild0 dataset are shown per nucleotide position as line plot (smoothened over 4 neighbors). The grey area marks the IGH VDJ junction. B.-E. like (A) but with different permutations as indicated. The remaining permutations are displayed in the supplementary Figure 2. 
categorized as "productive with detected indels" unfavorable.

\section{HTS of hybridoma ssUID libraries}

Next, the IMGT HighV-QUEST tool and a PGMtailored data processing pipeline developed by our group were tested using real HTS datasets derived from 7 monoclonal hybridomas (Figure 1). The HTS libraries were prepared using an IonTorrent PGM tailored single-side UID approach (supplementary Figure 3) allowing for error correction through building consensus sequences from all reads within a UID family [33, 34]. The ssUID barcodes, together with the C-region primer and appropriate 'GATC' spacer, were correctly identified at the sequencing start site of $99.12 \% \pm 0.56 \%$ of the usable reads containing a sample specific MID (Table 1). Between 146,010 and 739,854 reads were obtained per sample, with varying SsUID family size distributions (Figure 5A). After raw data processing, 1,431 to 47,169 consensus sequences were retained per hybridoma (Table 1) and uploaded to IMGT HighV-QUEST.

\section{IMGT HighV-QUEST processing of HTS hybridoma datasets}

The majority of the sequences returned by IMGT HighV-QUEST were categorized as productive $(75.8 \%$ $\pm 22.6 \%)$ and $10.9 \%( \pm 9.6 \%)$ were categorized as productive with detected indels (Table 2). The remaining sequences were either categorized as unproductive or unknown/else. To investigate the undetected or uncorrected errors within the two productive categories, sequences were aligned to their corresponding references. For Hybridoma 3, which had the poorest UID distribution (Figure 5A), only $26.8 \%$ of the sequences were classified as productive and $68.8 \%$ unproductive (Table 2 ). This hybridoma was therefore excluded from further analysis.
In the group of productive sequences with detected errors, the IMGT HighV-QUEST indel correction algorithm improved the number of correct sequences by $54.1 \%$ to on average $55.3 \%( \pm 32.0 \%$, Figure $5 \mathrm{~B})$. As expected, IMGT HighV-QUEST corrected most sequences that contained single insertions efficiently, reducing these errors from average $25.2 \%( \pm 24.3 \%)$ to $0.48 \%( \pm 0.72 \%, p$-value $=0.0027$, two-tailed $t$-test in Graphpad Prism, using Holm-Sidak's method [35] to account for multiple testing with alpha $=5 \%$, Figure $5 \mathrm{~B}$ ). Single deletions were found at somewhat higher rates than single insertions $(29.9 \% \pm 24.3 \%)$ of the sequences. They increased slightly after IMGT HighV-QUEST error correction $(31.6 \% \pm 24.1 \%)$, as insertions of higher indel permutations were corrected, leaving only deletions in the sequences. Accordingly, these higher permutations were found in $33.8 \%( \pm 23.8 \%)$ of the sequences before error-correction and reduced to $8.8 \%( \pm 6.3 \%)$ afterwards. While the detection of indel errors in the sequences by IMGT HighV-QUEST was efficient, the remaining errors after correction still affected $44.7 \% \pm 32.2 \%$ of the sequences. As described for the benchmarking sequences above, this makes further consideration of sequences marked as "productive with detected indels" inadvisable.

Sequences marked as productive without detected indels are not modified by IMGT HighV-QUEST but can nonetheless contain indel and nucleotide substitution errors. IMGT HighV-QUEST does not detect ambiguous nucleotides as errors but marks them as silent mutations. On average $2.2 \%( \pm 1.6 \%)$ of the consensus sequences in the productive dataset without detected indels contained ambiguous nucleotides (Table 3), which were discarded from the datasets. Most of the remaining sequences were indeed error-free $(98.8 \% \pm 0.5 \%$, Figure $5 \mathrm{C})$. The other $1.2 \%$ contained on average $0.2 \%( \pm 0.1 \%)$ ild 1 indels in close proximity to each other, masking frameshifts. Some sequences showed single insertions $(0.1 \% \pm 0.2 \%)$ and deletions $(0.15 \% \pm 0.13 \%)$, either at the beginning or the end, without detectable frameshift. The remaining

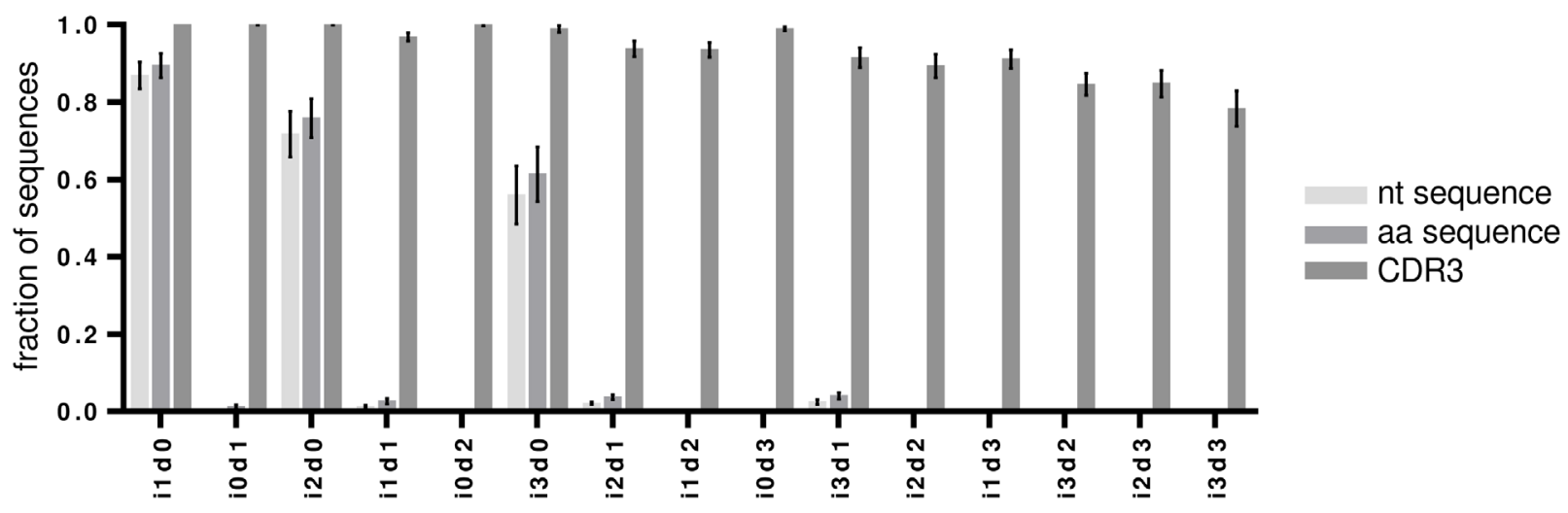

Figure 4: Correction of artificially introduced indels by IMGT HighV-QUEST. The fraction of correct sequences after IMGT HighV-QUEST processing for each artificial indel permutation are shown as bar charts for nucleotide (nt), amino acid (aa) and CDR3 amino acid sequences. Error bars indicate SD over all 7 artificial datasets. 
false sequences contained nucleotide substitutions, with the majority being transversions $(0.5 \% \pm 0.3 \%)$ and very few transitions $(<0.1 \%)$. As described by Shugay and coworkers, such substitutions originate from dominating polymerase errors occurring early during the amplification
[33]. As polymerase errors are occurring at relatively random positions, it is stochastically unlikely, that the same errors are found repeatedly within a dataset and can thus be accounted for by considering only consensus sequences that appear more than once in the final dataset
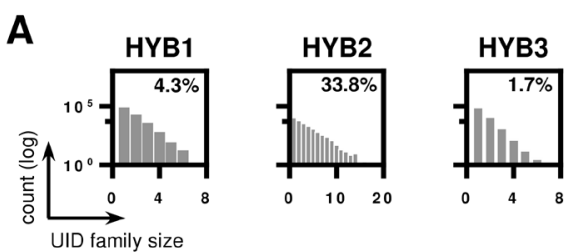

B

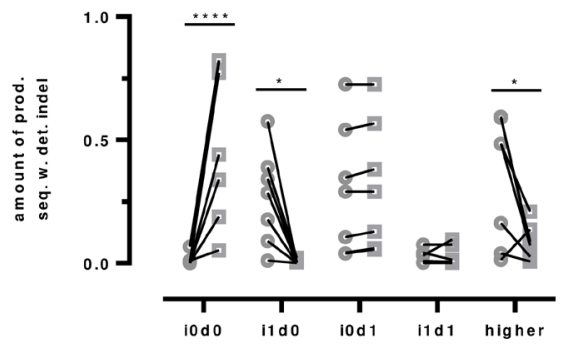

D
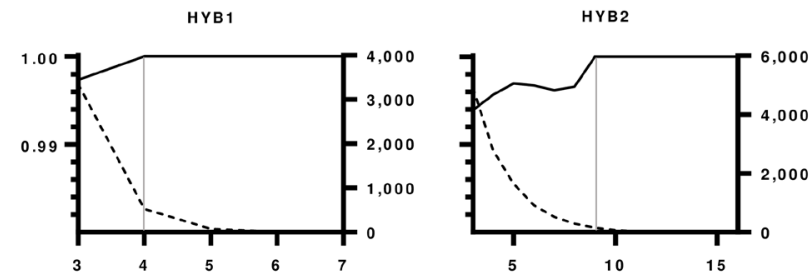

HYB5
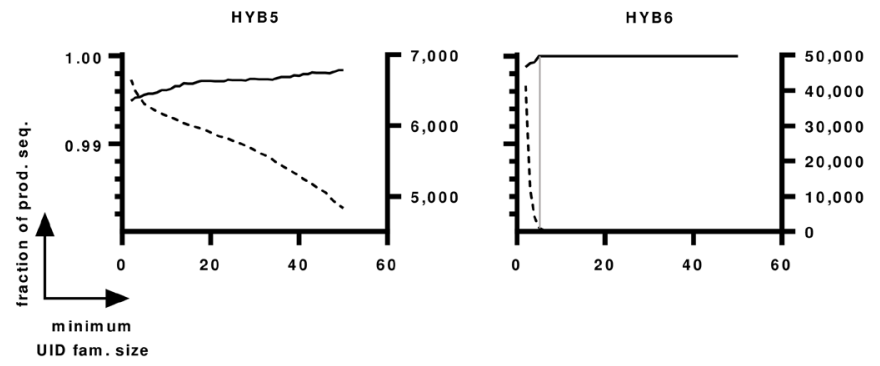
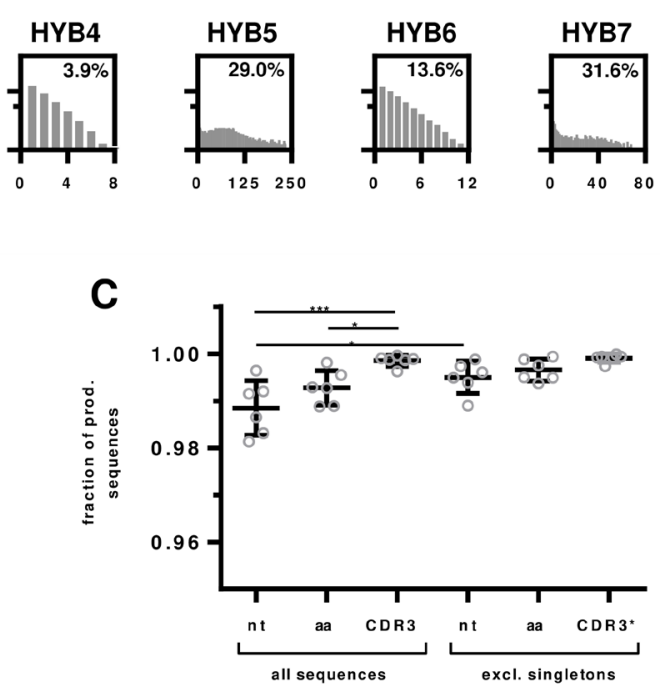

Figure 5: HTS data of monoclonal hybridomas libraries. A. UID family size distributions per sample. The number of UID families (log transformed) is plotted by the number of reads assigned to a ssUID per hybridoma. The amount of UID families containing a minimum of 3 reads are indicated as percentage value. B. Indel distributions on productive sequences with detected errors before and after IMGT HighV-QUEST processing. The amount of indel-free (i0d0), single insertions (i1d0), single deletions (i0d1), one single insertion and deletion (ild1) and higher permutations ("higher") are shown as fraction of productive reads with detected indels before (circles) and after (squares) IMGT HighV-QUEST error correction. Statistical differences are indicated with **** $p<0.0001, * p<0.05$ determined by multiple two-tailed $t$-test with Holm-Sidak's method to account for multiple testing. C. The influence of removing singletons on the number of error-free sequences in the productive dataset. The fractions of total sequences without detected indels are shown as boxplot with mean and \pm SD. Data are shown for all nucleotide sequences (nt), amino acid sequences (aa) and CDR3s for all sequences and data without singleton sequences. CDR3* indicates that for this set, singletons were determined on the full-length amino acid sequences. P values are indicated $* * * p<0.001, *<0.05$, One-way ANOVA with Sidak's post-hoc test. All other differences were not statistically significant. D. Influence of UID family size on the number of correct sequences. The number of correct sequences are shown as black line per minimum UID family size (left y-axis). The number of consensus sequences are shown as dotted line per minimum UID family size (right y-axis). The UID family size at which all sequences are correct is indicated by a grey vertical line for Hybridoma 1,2,4,6 and 7, the dataset of Hybridoma 5 does not reach $100 \%$ correct sequences. 
Table 4: HTS BM datasets classifications by IMGT HighV-QUEST

\begin{tabular}{|l|l|l|l|l|l|l|l|l|}
\hline Set & prod. seq. & $\mathbf{\%}$ & prod. w. det. indel & $\mathbf{\%}$ & unprod & $\mathbf{\%}$ & unknown/ else & \% \\
\hline BM1 & 30,003 & $79.2 \%$ & 5,585 & $14.7 \%$ & 1,015 & $2.7 \%$ & 1,274 & $3.4 \%$ \\
\hline BM2 & 30,971 & $82.8 \%$ & 5,214 & $13.9 \%$ & 999 & $2.7 \%$ & 204 & $0.5 \%$ \\
\hline BM3 & 27,990 & $85.5 \%$ & 3,777 & $11.5 \%$ & 776 & $2.4 \%$ & 205 & $0.6 \%$ \\
\hline BM4 & 28,371 & $73.4 \%$ & 8,747 & $22.6 \%$ & 1,241 & $3.2 \%$ & 276 & $0.7 \%$ \\
\hline Mean & 29,335 & $80,2 \%$ & 5,832 & $15,7 \%$ & 490 & $1.3 \%$ & 1,008 & $2.7 \%$ \\
\hline SD & 1,210 & $4.5 \%$ & 1,814 & $4,2 \%$ & 454 & $1.2 \%$ & 165 & $0.3 \%$ \\
\hline
\end{tabular}

$[33,34]$. Following this approach, the data was reassessed, excluding singleton consensus sequences. This reduced the number of total sequences in the datasets by $0.8 \%$ $( \pm 0.4 \%)$. The number of transversions was reduced significantly by $0.3 \%$ to $0.16 \%( \pm 0.19 \%, p$-value $=0.008$, two-tailed $t$-test in Graphpad Prism, using Holm-Sidak's method to account for multiple testing with alpha $=5 \%$, data not shown). Consequently, the number of error-free sequences improved significantly by $0.7 \%$ to $99.5 \%$ ( \pm $0.3 \%, p$-value $<0.0001$, two-tailed $t$-test, using HolmSidak's method to account for multiple testing with alpha $=5 \%$ ).

The number of reads per UID, referred to as UID family size, is crucial to obtain reliable consensus sequences [34]. Increasing the minimum number of required reads per UID family improved the amount of correct sequences, reaching $100 \%$ for all hybridomas, except Hybridoma 5, albeit with different UID family sizes (Figure 5D). However, with increasing minimum UID family sizes, the number of sequences decreased exponentially. Consequently, at the point of reaching $100 \%$ correct sequences, on average only $7.9 \%( \pm 7.1 \%$, excl. Hybridoma 5) of the sequences remained (Figure 5D). According to our data, keeping a minimum UID family size of 3 provided adequate accuracy and throughput when using an IonTorrent PGM. As expected, the number of correct amino acid sequences was higher $(99.3 \% \pm 0.3 \%)$ than the amount of correct nucleotide sequences (Figure $5 \mathrm{C})$. An average of $0.6 \%( \pm 0.4 \%)$ of the sequences was subject to amino acid changes. Excluding singleton amino acid sequences increased the number of correct amino acid sequences to $99.7 \%( \pm 0.2 \%)$, but this increase was not statistically significant. CDR3 amino acid sequences were returned almost entirely correct $(99.85 \% \pm 0.11 \%$, Figure $3 \mathrm{C})$, increasing to $99.91 \%$ ( $\pm 0.08 \%)$ when singleton fulllength amino acid sequences were excluded.

\section{HTS and processing of bulk mouse BM samples}

To verify our ssUID approach for bulk sequencing, mouse bone marrow samples from four mice were processed with $\mathrm{C}$-region specific primers targeting mouse IgG and IgM isotype BCRs (Supplementary Table 3). The ssUID barcodes, together with C-region primers and 'GATC' spacer, were identified at the sequencing start site of $89.1 \% \pm 2.1 \%$ of the usable reads containing a sample specific MID (Table 1). All samples had homogeneous ssUID family size distributions for both isotypes (Figure

A

B
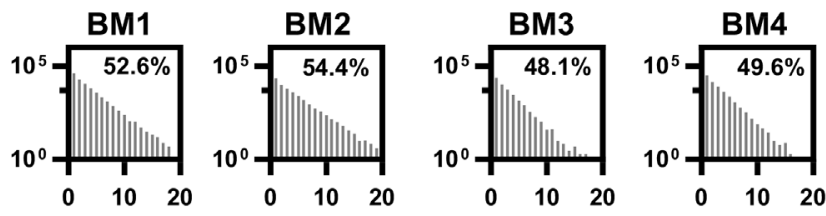

$\lg G$
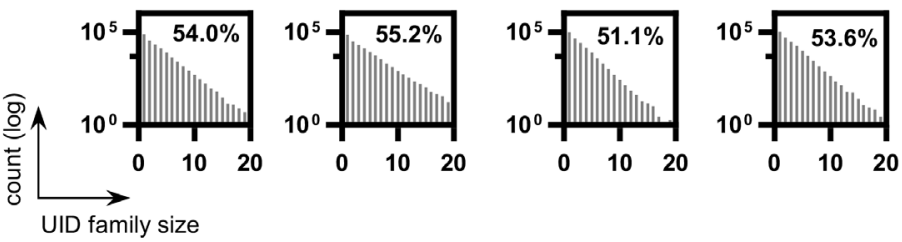

$\lg M$

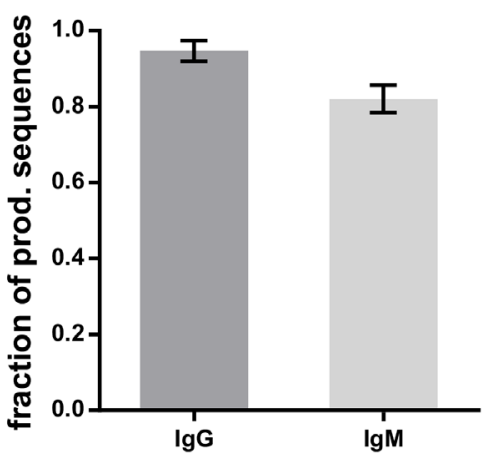

Figure 6: HTS data on bulk BM libraries. A. UID family size distributions per sample. The number of UID families (log transformed) is plotted by the number of reads assigned to a ssUID per bulk BM library. The amount of UID families containing a minimum of 3 reads are indicated as percentage value. B. The number of sequences retained after excluding singleton for bulk BM HTS datasets. Bars represent the fraction of total sequences after excluding singletons for IgG (grey) and IgM (light grey), respectively. Error bars indicate the SD over all four datasets. 
$6 \mathrm{~A})$. On average, $51.2 \%( \pm 2.5 \%)$ and $53.5 \%( \pm 1.5 \%)$ of the ssUID families for $\mathrm{IgG}$ and $\mathrm{IgM}$, respectively, contained more than two sequences and were considered for consensus building. This resulted in 5,170 to 12,025 consensus sequences were retained for IgG samples and 25,825 to 31,612 consensus sequences for the IgM isotype per isotype after raw data processing (Table 4) which were subsequently uploaded to IMGT HighV-QUEST.

IMGT HighV-QUEST returned $80.2 \%( \pm 4.5 \%)$ of the sequences as productive and $15.7 \%( \pm 4.2 \%)$ of the sequences contained indels, as detected by the IMGT HighV-QUEST indel identification algorithm (Table 4). On average, $2.7 \%( \pm 0.3 \%)$ of the sequences were found unproductive and the remaining were categorized as unknown/else. After IMGT HighV-QUEST processing, most of the IgG sequences were found with two or more copies $(94.7 \% \pm 2.4 \%)$ and as expected, less for the IgM sequences $(82.0 \% \pm 3.1 \%)$ (Figure $6 \mathrm{~B})$. As determined above, these singlet sequences should be removed from final data to obtain highly reliable sequencing results.

\section{DISCUSSION}

Investigation of IG repertoires by HTS is challenging both with respect to the library preparation as well as sequencing error assessment and data processing. Using artificially falsified sequences, we show here that the IMGT HighV-QUEST indel detection algorithm is efficient while the IMGT HighV-QUEST indel correction algorithm only corrects single insertions sufficiently. We confirm the utility of the IonTorrent PGM to assess murine IGH repertoires with high confidence, using a dedicated library preparation protocol with a PGM-tailored $16 \mathrm{nt}$ single side unique identifier (ssUID) barcoding technique. Our data show that appropriate data processing reduced the error rate of PGM-sequenced IGH repertoires to less than $0.5 \%$ false nucleotide and amino acid sequences, and to less than $0.01 \%$ false CDR3 sequences per dataset.

Sequencing of IGH repertoires requires a thorough assessment and correction of platform inherent sequencing errors [7, 9, 12-15]. Using the IMGT HighV-QUEST tool for reference alignment, the indel errors of the utilized Ion Torrent PGM sequencing platform can theoretically be detected through the resulting codon frame-shifts [17]. The VDJ structure of the IGH sequence facilitates indel detection by frame-shift, since gene segments can be aligned separately. In our study, the IMGT HighVQUEST algorithm successfully detects $97.9 \%$ of all indels, regardless of their composition, only single insertions or deletions at the beginning or the end of the sequences $(7.9 \%$ and $7.5 \%$, respectively), or $\mathrm{i} 1 \mathrm{~d} 1$ compositions in close proximity to each other could not be identified (8.5\%). IMGT HighV-QUEST tries to correct detected insertions subsequently by removing the false nucleotide(s) according to the predicted germline sequence. In the artificially falsified datasets of our study insertion-only errors were corrected by the IMGT HighV-QUEST algorithm with 87\% (i1d0), 72\% (i2d0) and $56 \%$ (i3d0) efficiency. Deletions, on the other hand, are more difficult to recover since the missing nucleotide cannot necessarily be inferred from the germline sequence with sufficient confidence. Consequently, artificially introduced deletions were not corrected by IMGT HighVQUEST. Also, for sequences with mixed insertions and deletions only the nucleotide insertions were corrected by IMGT HighV-QUEST leaving the sequence erroneous. Furthermore, indels could impair the alignment process by changing the identification of the closest germline. While we observed more potential germlines to be suggested by IMGT HighV-QUEST for the higher indel permutations, the selected assignment by IMGT HighVQUEST of the closest germline did not change even with the highest permutation of indels (i3d3) tested (data not shown). Taken together, these data indicate that detection of indels by IMGT HighV-QUEST is highly efficient and sequences categorized as "productive" without detected errors are almost entirely indel-free. The low efficiency of the indel correction algorithm makes it inadvisable to take productive sequences with detected indels into account for any downstream analysis. These correspond to about $10 \%$ of the final HTS consensus sequences in our study.

HTS library preparation using multiple primers during template amplification can significantly bias the repertoire composition $[14,19]$. This bias is essentially removed by UID barcoding, but the approach reduces sequencing depth at the same time [34, 36-38]. In our study, the raw sequencing depth does not influence the relative number of correct sequences while the average UID family size proved to be crucial. For instance, Hybridoma 3, although having only the $4^{\text {th }}$ lowest amount of raw-reads, lacked eligible UID family sizes $(>2$ sequences per UID). For Hybridoma 3, less than $1.7 \%$ of the UID families had more than 2 members, resulting in the poorest error correction rate during sample processing, potentially because of low amounts of IGH encoding mRNA molecules. While the UID family sizes for the presented Hybridoma sequencing datasets vary largely, the bulk sequencing experiment generated homogeneous datasets with around $50 \%$ eligible reads. We were unable to determine the original cause of the poor performance of Hybridoma 3 for HTS which demonstrates, that it is crucial to critically follow samples throughout the entire raw data processing. As for this Hybridoma 3, it became evident that the dataset had low quality only after IMGT HighVQUEST processing, returning just $26.6 \%$ of the consensus sequences as productive. Datasets of higher complexity than single sequence Hybridoma libraries could be even more elusive. We thus conclude from our data, that for applying a UID family-wise consensus building approach, samples with low numbers of eligible consensus reads after pre-IMGT HighV-QUEST processing or unusually high numbers of unproductive sequences $(>$ number 
of productive sequences) post-IMGT HighV-QUEST alignment should be discarded from further analysis.

For grouping reads by UID families, it is essential to identify the UID tags correctly $[34,38]$. The PGM sequencing chemistry is unidirectional, starting with the sequencing adapter A. Comparable protocols for the Illumina sequencing platforms usually consist of UID tags at the beginning and the end of the amplicon sequence [39]. We chose to introduce the 16 random nucleotides of the UID tag at the sequencing start site as the PGM semiconductor technology is significantly less accurate towards the end of the sequence [40]. We included a 4-nucleotide spacer as junction into the UID tag resulting in the $\mathrm{N}_{8}$-GATC- $\mathrm{N}_{8}$ ssUID layout of this study. Like this, we address that the PGM indel rate increases in homopolymer stretches with their length [41], in particular when homopolymers are longer than 8nt [42]. While breaking potential homopolymer patterns within the UID, this design also reduces the number of mistakes during primer synthesis and allows to generate sets of primers with individual spacers that could be used to tag different experiments.

Nucleotide substitution errors are the most difficult to account for in HTS IG repertoire approaches and can critically falsify somatic hypermutation profiles [16, 24]. They can originate from mixed events of adjacent insertions and deletions, which cannot be detected by the IMGT HighV-QUEST algorithm or are introduced as mistakes by the sequencing platform. UID barcoded RNA transcripts allow us to address this problem $[8,33,34$, 39]. B cells contain up to several thousands of identical IG RNA molecules that are each individually tagged by a UID $[39,43]$. Therefore, a HTS run provides a snapshot of the relative abundance of RNA transcripts [16]. Comparable to procedures used for identification of single nucleotide polymorphisms (SNP), single occurrences of nucleotide substitutions can be ruled out as artifacts and only transcripts above a certain copy threshold should be retained [43]. Our data show that considering sequences with at least 2 copies in the final dataset improves the proportion of correct sequences by $0.7 \%$ to $99.5 \%$ by reducing the number of sequences by merely $0.8 \%$. Compared to Hybridoma HTS datasets, more sequences are removed by this step from bulk BM IgG and IgM data, with IgM isotype exhibiting the strongest reduction of sequences (18\%). This is expected by the higher diversity of these samples, with IgM isotype BCR carrying B cells being mainly naïve cells with little clonal expansion. However, as the sequences in the datasets from monoclonal Hybridomas are all derived from identical RNA molecules, it makes it stochastically more likely, that the same indel error appears several times. Thus, it can be expected that excluding singletons would increase the number of correct sequences in the bulk B cell derived datasets even more, where less sequences are derived from identical RNA molecules.
For large scale HTS experiments spanning several treatment groups, it is essential to have a reliable library preparation protocol resulting in sequencing libraries with similar depth and limited variation to reduce potential batch effects. The presented workflow returned very homogeneous datasets for libraries prepared from bulk murine BM samples. Even with both isotype primers applied together during reverse transcription, the BM samples showed comparable numbers of reads and sequences throughout the entire data processing approach with little variation. Approximately half of the sequences are lost because they belong to ssUID-families with only a single member. As determined through HTS of the monoclonal hybridoma libraries, those sequences are not reliable and thus should be excluded from further processing. We tried to increase the number of eligible ssUID families by increasing the number of PCR cycles in the amplification step but found that it reduced overall sequencing depth as average family size increased drastically (data not shown). Overall, the presented workflow generates robust and homogeneous data for bulk sequencing approaches of murine BM samples.

In conclusion, we have demonstrated that using our ssUID library preparation in combination with the IMGT database, the PGM sequencing platform can be efficiently used to assess murine IGH repertoires. Considering only consensus sequences with at least two copies in the final dataset improved the sequence quality considerably. Taken together, this approach allowed to obtain highly reliable IGH sequences, with more than $99 \%$ confidence in general and $99.9 \%$ confidence for the correct CDR3 sequences. The protocol and sample processing strategies described in this study will help to establish the benchtop-scale Ion Torrent sequencing technology of animal models in the field of immunoglobulin repertoire research.

\section{MATERIALS AND METHODS}

\section{Animals}

All animal procedures were in compliance with the rules described in the Guide for the Care and Use of Laboratory Animals and accepted by the 'Comité National d'Éthique de Recherche' (CNER, Luxembourg). Balb/c mice (10-week old, female) were obtained from Harlan (Horst, NL) and acclimatized for 1 week. Animals were kept under timed $12 \mathrm{~h} \mathrm{light/dark} \mathrm{cycles} \mathrm{at} 22{ }^{\circ} \mathrm{C}$ and $40 \%$ relative humidity with food and water available ad libitum.

\section{RNA extraction}

RNA was extracted with Trizol LS/chloroform (Thermo Fisher Scientific, Waltham, USA) method from seven monoclonal hybridoma cell lines (produced 
from Balb/C mice in house) with $10^{6}$ cells each. DNA was digested using the DNAfree kit (Thermo Fisher Scientific), RNA was further purified using Agencourt ${ }^{\circledR}$ RNAclean XP beads (Analis, Suarlée, BE) and quantified on a NanoDrop ${ }^{\circledR}$ Spectrophotometer (ND1000, Isogen Life Science, De Meern, NL). RNA was either directly used for library preparation or stored at $-80^{\circ} \mathrm{C}$. For bulk bone marrow samples, lymphocytes were first isolated by density-gradient centrifugation (ficoll ${ }^{\circledR}$ Paque Plus, SigmaAldrich) from bone marrow washes. Samples were then processed the same way as for the hybridomas.

\section{Reference sequences}

Hybridoma cDNA transcripts were obtained using mouse constant region IgG primer (Supplementary Table 3) in a Superscript III (Thermo Fisher Scientific) reverse transcription following the manufacturer's instructions for templates with high GC content. Transcripts were Sangersequenced (3100 Avant, Thermo Fisher Scientific) using constant region $\mathrm{IgG}$ and V-region primers (Supplementary Table 3). Forward and reverse sequences were aligned and submitted to IMGT V-QUEST (http://www.imgt.org, [44]) to verify the nucleotide sequence and to translate into amino acids. These sequences were subsequently used as reference sequences in alignments and artificial error insertion experiments.

\section{Datasets with artificial insertions and deletions}

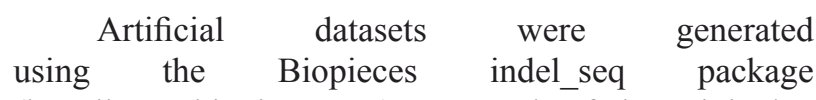
(http://www.biopieces.org). For each of the original 7 hybridoma sequences, 2500 error-containing sequences were generated by combining 0-3 insertions and 0-3 deletions, obtaining a total of 37500 artificial sequences per hybridoma. For every set, indel-type and -position were determined by alignment to the original sequence to ensure homogenous error distributions. All artificial datasets were uploaded to IMGT HighV-QUEST and sorted by annotation: IMGT HighV-QUEST annotates correct sequences as productive. Sequences with a detected indel (frameshift, stop codon) are marked as "productive (see comment)" if the error can be corrected (referred to as "productive with detected errors"). Sequences with uncorrectable errors are classified as "unproductive". If no fitting germline can be found sequences are marked as "unknown" or "no result" (referred to here as "unknown/ else"). The remaining indels on nucleotide level and amino acid changes were determined using the SeqAn library [45] in a custom-made $\mathrm{C}++$ reference alignment program. For datasets with one insertion and one deletion (i1d1) the positions of the indels were determined by positionwise mismatch detection using a custom made Biopython [46] script. Upon detection, the nucleotide positions were returned, and the process repeated with reverse complement sequences.

\section{Library preparation and HTS}

Approximately 100ng (as determined by Nanodrop ${ }^{\circledR}$ ) of total RNA per hybridoma or bone marrow was used for library preparation. We adapted the UID labeling method developed by Vollmers et al [39] to our PGM sequencing system (supplementary Figure 3). RNA was reverse transcribed using Superscript III reverse transcriptase, according to the manufacturer's instructions, using multiplex identifiers (MID) and UID tagged mouse constant region ( $\mathrm{IGH} \gamma$ ) primers elongated by partial PGM sequencing adapter pA (Supplementary Table 3). The MID tag allowed multiplexing of several samples on one sequencing chip. The UID tag consists of two times 8 random nucleotides separated by a "GATC" spacer $\left(\mathrm{N}_{8}\right.$ GATC-N ${ }_{8}$ ). With this UID tag each RNA molecule targeted by the primer is uniquely labeled (see $[33,39]$ for detailed theoretical descriptions). The RT reaction mixtures were split into two equal second strand synthesis reactions using Phusion ${ }^{\circledR}$ High-Fidelity DNA polymerase (NEB, Massachusetts, USA) with a mouse IGH V-region primer mix (Supplementary Table 3 ). The reaction conditions were as follows: $98^{\circ} \mathrm{C} 2 \mathrm{~min}, 50^{\circ} \mathrm{C} 2 \mathrm{~min}, 72^{\circ} \mathrm{C} 10 \mathrm{~min}$ in a single cycle reaction. Both reaction aliquots were combined and purified twice using Agencourt ${ }^{\circledR}$ AMPure ${ }^{\circledR}$ XP beads (Analis) in a $1: 1(\mathrm{v} / \mathrm{v})$ ratio to remove primer traces. Libraries were subsequently amplified with a Q5 ${ }^{\circledR}$ Hot Start High-Fidelity DNA polymerase (NEB) using the full-length Ion Torrent PGM sequencing adapters A and P1 as primers (Supplementary Table 3 ) with the following conditions: $98^{\circ} \mathrm{C}$ for $1 \mathrm{~min}, 20$ cycles of $98^{\circ} \mathrm{C}$ for $10 \mathrm{~s}, 65^{\circ} \mathrm{C}$ for $20 \mathrm{~s}, 72^{\circ} \mathrm{C}$ for 30 seconds. Final elongation was done at $72^{\circ} \mathrm{C}$ for $2 \mathrm{~min}$. Amplified libraries were purified twice using equal volumes of AMPure ${ }^{\circledR}$ XP beads. Quality of the libraries as well as size of the amplicon and concentrations were determined using Agilent 2100 Bioanalyzer (Agilent Technologies, Diegem, BE) with the High Sensitivity DNA Kit (Agilent Technologies). 10 libraries were pooled equimolar on an Ion $316^{\mathrm{TM}}$ Chip (Thermo Fisher Scientific) and sequenced on a PGM sequencer, with all quality trimming options disabled on the Torrent Suite ${ }^{\mathrm{TM}}$ V4.0.2.

\section{Data processing pipeline for the HTS datasets}

Untrimmed raw reads were demultiplexed by their MIDs, retaining only sequences containing the full UID primer sequence for further analysis, with no mismatches allowed. The UID sequence was extracted and categorized in relation to the starting position of the detected primer including the GATC spacer and stored in the sequence identifier. After clipping the MID, UID and constant region primer, the trimmed reads were 
quality controlled ( $80 \%$ of the bases Phred-like quality score above 20) and grouped into UID families. Using pagan-msa [47], a consensus sequence was generated for each UID-family containing more than 2 members. Afterwards, reverse primers were identified with up to 2 mismatches and clipped. Subsequently, sequences were collapsed to unique reads, storing counts in the read identifier, and uploaded to IMGT HighV-QUEST for error detection, correction, annotation and translation into amino acids. Post-IMGT HighV-QUEST datasets were separated into four categories ("productive", "productive with detected errors", "unproductive" and "unknown/ else") and processed separately. Data processing was performed using custom-made Python scripts (Python v2.7) employed in a parallelizing bash wrapper script using gnu-parallel [48] and the Biopieces framework (http://www.biopieces.org/).

\section{Graphs and statistics}

All graphs and statistical analyses were performed using R base packages or GraphPad Prism 6. Average numbers are reported as mean \pm standard deviation (SD) unless specified otherwise.

\section{Abbreviations}

BM - Bone marrow; CDR3 - complementary determining region 3; HTS - high-throughput sequencing; IG - immunoglobulin; IGH - immunoglobulin heavy chain; IMGT - ImMunoGeneTics; indel - insertions and deletions of nucleotides; MID - multiplex identifier; nt - nucleotide; PGM - (Ion Torrent) Personal Genome Machine; UID - Unique (molecular) identifier; ssUID single side unique molecular identifier

\section{Author contributions}

J-P.B. designed research, cultivated hybridomas, performed library preparation, developed bioinformatics approaches, performed data processing, interpreted data and wrote the manuscript. W.J.F. and O.H. supported and developed bioinformatics approaches and performed data processing. D.E.M. prepared bulk sequencing libraries. A.R.S.X.D designed research, conducted animal experiments and interpreted data. A.W-B. developed and wrote the raw data processing bioinformatics pipeline. R.S. performed Ion Torrent PGM sequencing. A.B. designed research, supervised work, assisted library preparation and hybridoma cultivation and interpreted data C.P.M. supervised work, provided important intellectual input and interpreted data. All authors have read and corrected the manuscript.

\section{ACKNOWLEDGMENTS}

We would like to thank Josiane Kirpach for independently verifying the library preparation protocol and Fleur A.D. Leenen for critically revising the manuscript.

\section{CONFLICTS OF INTEREST}

The authors declare no conflict of interest.

\section{FUNDING}

J.-P. Bürckert and A.R.S.X. Dubois were supported by the AFR (Aides à la Formation Recherche) fellowships \#7039209 and \#1196376, respectively, from the FNR (Fonds National de la Recherche), Luxembourg.

\section{REFERENCES}

1. Tonegawa S. Somatic generation of antibody diversity. Nature. 1983; 302:575-81.

https://doi.org/10.1038/302575a0

2. Reddy ST, Georgiou G. Systems analysis of adaptive immunity by utilization of high-throughput technologies. Curr Opin Biotechnol. 2011; 22:584-89.

https://doi.org/10.1016/j.copbio.2011.04.015

3. Fischer N. Sequencing antibody repertoires: the next generation. MAbs. 2011; 3:17-20.

https://doi.org/10.4161/mabs.3.1.14169

4. Reddy ST, Ge X, Miklos AE, Hughes RA, Kang SH, Hoi KH, Chrysostomou C, Hunicke-Smith SP, Iverson BL, Tucker PW, Ellington AD, Georgiou G. Monoclonal antibodies isolated without screening by analyzing the variable-gene repertoire of plasma cells. Nat Biotechnol. 2010; 28:965-69. https://doi.org/10.1038/nbt.1673

5. Boyd SD, Marshall EL, Merker JD, Maniar JM, Zhang LN, Sahaf B, Jones CD, Simen BB, Hanczaruk B, Nguyen KD, Nadeau KC, Egholm M, Miklos DB, et al. Measurement and clinical monitoring of human lymphocyte clonality by massively parallel VDJ pyrosequencing. Sci Transl Med. 2009; 1:12ra23.

https://doi.org/10.1126/scitranslmed.3000540

6. Weinstein JA, Jiang N, White RA 3rd, Fisher DS, Quake SR. High-throughput sequencing of the zebrafish antibody repertoire. Science. 2009; 324:807-10.

https://doi.org/10.1126/science. 1170020

7. Metzker ML. Sequencing technologies - the next generation. Nat Rev Genet. 2010; 11:31-46.

https://doi.org/10.1038/nrg2626

8. He L, Sok D, Azadnia P, Hsueh J, Landais E, Simek M, Koff WC, Poignard P, Burton DR, Zhu J. Toward a more accurate view of human B-cell repertoire by next- 
generation sequencing, unbiased repertoire capture and single-molecule barcoding. Sci Rep. 2014; 4:6778.

https://doi.org/10.1038/srep06778

9. Huse SM, Huber JA, Morrison HG, Sogin ML, Welch DM. Accuracy and quality of massively parallel DNA pyrosequencing. Genome Biol. 2007; 8:R143.

https://doi.org/10.1186/gb-2007-8-7-r143

10. Nguyen P, Ma J, Pei D, Obert C, Cheng C, Geiger TL. Identification of errors introduced during high throughput sequencing of the $\mathrm{T}$ cell receptor repertoire. BMC Genomics. 2011; 12:106.

https://doi.org/10.1186/1471-2164-12-106

11. Fuellgrabe MW, Herrmann D, Knecht H, Kuenzel S, Kneba M, Pott C, Brüggemann M. High-Throughput, AmpliconBased Sequencing of the CREBBP Gene as a Tool to Develop a Universal Platform-Independent Assay. PLoS One. 2015; 10:e129195.

https://doi.org/10.1371/journal.pone.0129195

12. Zhu J, O'Dell S, Ofek G, Pancera M, Wu X, Zhang B, Zhang Z, Mullikin JC, Simek M, Burton DR, Koff WC, Shapiro L, Mascola JR, Kwong PD, and NISC Comparative Sequencing Program. Somatic populations of PGT135137 HIV-1-neutralizing antibodies identified by 454 pyrosequencing and bioinformatics. Front Microbiol. 2012; 3:315. https://doi.org/10.3389/fmicb.2012.00315

13. Deng W, Maust BS, Westfall DH, Chen L, Zhao H, Larsen BB, Iyer S, Liu Y, Mullins JI. Indel and Carryforward Correction (ICC): a new analysis approach for processing 454 pyrosequencing data. Bioinformatics. 2013; 29:240209. https://doi.org/10.1093/bioinformatics/btt434

14. Baum PD, Venturi V, Price DA. Wrestling with the repertoire: the promise and perils of next generation sequencing for antigen receptors. Eur J Immunol. 2012; 42:2834-39. https://doi.org/10.1002/eji.201242999

15. Bolotin DA, Mamedov IZ, Britanova OV, Zvyagin IV, Shagin D, Ustyugova SV, Turchaninova MA, Lukyanov S, Lebedev YB, Chudakov DM. Next generation sequencing for TCR repertoire profiling: platform-specific features and correction algorithms. Eur J Immunol. 2012; 42:3073-83. https://doi.org/10.1002/eji.201242517

16. Georgiou G, Ippolito GC, Beausang J, Busse CE, Wardemann H, Quake SR. The promise and challenge of high-throughput sequencing of the antibody repertoire. Nat Biotechnol. 2014; 32:158-68.

https://doi.org/10.1038/nbt.2782

17. Zhu J, Ofek G, Yang Y, Zhang B, Louder MK, Lu G, McKee K, Pancera M, Skinner J, Zhang Z, Parks R, Eudailey J, Lloyd KE, et al, and NISC Comparative Sequencing Program. Mining the antibodyome for HIV-1neutralizing antibodies with next-generation sequencing and phylogenetic pairing of heavy/light chains. Proc Natl Acad Sci USA. 2013; 110:6470-75.

https://doi.org/10.1073/pnas.1219320110

18. Carlson CS, Emerson RO, Sherwood AM, Desmarais
C, Chung MW, Parsons JM, Steen MS, LaMadridHerrmannsfeldt MA, Williamson DW, Livingston RJ, Wu D, Wood BL, Rieder MJ, Robins H. Using synthetic templates to design an unbiased multiplex PCR assay. Nat Commun. 2013; 4:2680.

https://doi.org/10.1038/ncomms3680

19. Khan TA, Friedensohn S, Gorter de Vries AR, Straszewski J, Ruscheweyh HJ, Reddy ST. Accurate and predictive antibody repertoire profiling by molecular amplification fingerprinting. Sci Adv. 2016; 2:e1501371-1501371. https://doi.org/10.1126/sciadv.1501371

20. Best K, Oakes T, Heather JM, Shawe-Taylor J, Chain B. Computational analysis of stochastic heterogeneity in PCR amplification efficiency revealed by single molecule barcoding. Sci Rep. 2015; 5:14629.

https://doi.org/10.1038/srep14629

21. Cocquet J, Chong A, Zhang G, Veitia RA. Reverse transcriptase template switching and false alternative transcripts. Genomics. 2006; 88:127-31.

https://doi.org/10.1016/j.ygeno.2005.12.013

22. Fu GK, Wilhelmy J, Stern D, Fan HC, Fodor SP. Digital encoding of cellular mRNAs enabling precise and absolute gene expression measurement by single-molecule counting. Anal Chem. 2014; 86:2867-70.

https://doi.org/10.1021/ac500459p

23. Choi NM, Loguercio S, Verma-Gaur J, Degner SC, Torkamani A, Su AI, Oltz EM, Artyomov M, Feeney AJ. Deep sequencing of the murine $\mathrm{IgH}$ repertoire reveals complex regulation of nonrandom $\mathrm{V}$ gene rearrangement frequencies. J Immunol. 2013; 191:2393-402.

https://doi.org/10.4049/jimmunol.1301279

24. Yaari G, Kleinstein SH. Practical guidelines for B-cell receptor repertoire sequencing analysis. Genome Med. 2015; 7:121. https://doi.org/10.1186/s13073-015-0243-2

25. Alamyar E, Duroux P, Lefranc MP, Giudicelli V. IMGT(®) tools for the nucleotide analysis of immunoglobulin (IG) and $\mathrm{T}$ cell receptor (TR) V-(D)-J repertoires, polymorphisms, and IG mutations: IMGT/V-QUEST and IMGT/HighV-QUEST for NGS. Methods Mol Biol. 2012; 882:569-604.

https://doi.org/10.1007/978-1-61779-842-9_32

26. Lefranc MP, Giudicelli V, Duroux P, Jabado-Michaloud J, Folch G, Aouinti S, Carillon E, Duvergey H, Houles A, Paysan-Lafosse T, Hadi-Saljoqi S, Sasorith S, Lefranc G, Kossida S. IMGT®, the international ImMunoGeneTics information system ${ }^{\circledR} 25$ years on. Nucleic Acids Res. 2015; 43:D413-22. https://doi.org/10.1093/nar/gku1056

27. van Dongen JJ, Langerak AW, Brüggemann M, Evans PA, Hummel M, Lavender FL, Delabesse E, Davi F, Schuuring E, García-Sanz R, van Krieken JH, Droese J, González D, et al. Design and standardization of PCR primers and protocols for detection of clonal immunoglobulin and T-cell receptor gene recombinations in suspect lymphoproliferations: report of the BIOMED-2 Concerted Action BMH4-CT98-3936. 
Leukemia. 2003; 17:2257-317.

https://doi.org/10.1038/sj.leu.2403202

28. Mestas J, Hughes CC. Of mice and not men: differences between mouse and human immunology. J Immunol. 2004; 172:2731-38. https://doi.org/10.4049/jimmunol.172.5.2731

29. Simonetti G, Bertilaccio MT, Ghia P, Klein U. Mouse models in the study of chronic lymphocytic leukemia pathogenesis and therapy. Blood. 2014; 124:1010-19. https://doi.org/10.1182/blood-2014-05-577122

30. Schroeder HW Jr. Similarity and divergence in the development and expression of the mouse and human antibody repertoires. Dev Comp Immunol. 2006; 30:11935. https://doi.org/10.1016/j.dci.2005.06.006

31. Hildebrand MV. The birthday problem. Am Math Mon. 1993; 100:643

32. Yousfi Monod M, Giudicelli V, Chaume D, Lefranc MP. IMGT/JunctionAnalysis: the first tool for the analysis of the immunoglobulin and $\mathrm{T}$ cell receptor complex V-J and V-D-J JUNCTIONs. Bioinformatics. 2004 (Suppl 1); 20:i379-85. https://doi.org/10.1093/bioinformatics/bth945

33. Shugay M, Britanova OV, Merzlyak EM, Turchaninova MA, Mamedov IZ, Tuganbaev TR, Bolotin DA, Staroverov DB, Putintseva EV, Plevova K, Linnemann C, Shagin D, Pospisilova S, et al. Towards error-free profiling of immune repertoires. Nat Methods. 2014; 11:653-55.

https://doi.org/10.1038/nmeth.2960

34. Turchaninova MA, Davydov A, Britanova OV, Shugay M, Bikos V, Egorov ES, Kirgizova VI, Merzlyak EM, Staroverov DB, Bolotin DA, Mamedov IZ, Izraelson M, Logacheva MD, et al. High-quality full-length immunoglobulin profiling with unique molecular barcoding. Nat Protoc. 2016; 11:1599-616.

https://doi.org/10.1038/nprot.2016.093

35. Holm S. A simple sequentially rejective multiple test procedure. Scand J Stat. 1979; 6:65-70.

http://www.jstor.org/stable/4615733

36. Shiroguchi K, Jia TZ, Sims PA, Xie XS. Digital RNA sequencing minimizes sequence-dependent bias and amplification noise with optimized single-molecule barcodes. Proc Natl Acad Sci USA. 2012; 109:1347-52. https://doi.org/10.1073/pnas.1118018109

37. Mamedov IZ, Britanova OV, Zvyagin IV, Turchaninova MA, Bolotin DA, Putintseva EV, Lebedev YB, Chudakov DM. Preparing unbiased T-cell receptor and antibody cDNA libraries for the deep next generation sequencing profiling. Front Immunol. 2013; 4:456.

https://doi.org/10.3389/fimmu.2013.00456

38. Egorov ES, Merzlyak EM, Shelenkov AA, Britanova OV, Sharonov GV, Staroverov DB, Bolotin DA, Davydov AN, Barsova E, Lebedev YB, Shugay M, Chudakov DM. Quantitative profiling of immune repertoires for minor lymphocyte counts using unique molecular identifiers. J Immunol. 2015; 194:6155-63. https://doi.org/10.4049/jimmunol.1500215

39. Vollmers C, Sit RV, Weinstein JA, Dekker CL, Quake SR. Genetic measurement of memory B-cell recall using antibody repertoire sequencing. Proc Natl Acad Sci USA. 2013; 110:13463-68.

https://doi.org/10.1073/pnas.1312146110

40. Loman NJ, Misra RV, Dallman TJ, Constantinidou C, Gharbia SE, Wain J, Pallen MJ. Performance comparison of benchtop high-throughput sequencing platforms. Nat Biotechnol. 2012; 30:434-39.

https://doi.org/10.1038/nbt.2198

41. Bragg LM, Stone G, Butler MK, Hugenholtz P, Tyson GW. Shining a light on dark sequencing: characterising errors in Ion Torrent PGM data. PLoS Comput Biol. 2013; 9:e1003031. https://doi.org/10.1371/journal.pcbi.1003031

42. Quail MA, Smith M, Coupland P, Otto TD, Harris SR, Connor TR, Bertoni A, Swerdlow HP, Gu Y, Rothberg J, Hinz W, Rearick T, Schultz J, et al. A tale of three next generation sequencing platforms: comparison of Ion Torrent, Pacific Biosciences and Illumina MiSeq sequencers. BMC Genomics. 2012; 13:341.

https://doi.org/10.1186/1471-2164-13-341

43. Kinde I, Wu J, Papadopoulos N, Kinzler KW, Vogelstein B. Detection and quantification of rare mutations with massively parallel sequencing. Proc Natl Acad Sci USA. 2011; 108:9530-35.

https://doi.org/10.1073/pnas.1105422108

44. Brochet X, Lefranc MP, Giudicelli V. IMGT/V-QUEST: the highly customized and integrated system for IG and TR standardized V-J and V-D-J sequence analysis. Nucleic Acids Res. 2008; 36:W503-8.

https://doi.org/10.1093/nar/gkn316

45. Iacobuzio-Donahue CA, Ashfaq R, Maitra A, Adsay NV, Shen-Ong GL, Berg K, Hollingsworth MA, Cameron JL, Yeo CJ, Kern SE, Goggins M, Hruban RH. Highly expressed genes in pancreatic ductal adenocarcinomas: a comprehensive characterization and comparison of the transcription profiles obtained from three major technologies. Cancer Res. 2003; 63:8614-22.

https://doi.org/10.1126/science.1058040

46. Cock PJ, Antao T, Chang JT, Chapman BA, Cox CJ, Dalke A, Friedberg I, Hamelryck T, Kauff F, Wilczynski B, De Hoon MJ. Biopython: Freely available Python tools for computational molecular biology and bioinformatics. Bioinformatics. Oxford University Press; 2009; 25: 1422-3. https://doi.org/10.1093/bioinformatics/btp163

47. Löytynoja A, Vilella AJ, Goldman N. Accurate extension of multiple sequence alignments using a phylogeny-aware graph algorithm. Bioinformatics. 2012; 28:1684-91.

https://doi.org/10.1093/bioinformatics/bts198

48. Tange O. GNU Parallel: the command-line power tool. USENIX Mag. 2011; 36:42-47.

https://doi.org/10.5281/zenodo. 16303 